\title{
Polarization experiments with antiproton beam
}

\author{
V. V. MOCHALOV ${ }^{1, a, b}$, P. A. SEMENOV ${ }^{a, b}$, M. N. STRIKHANOV ${ }^{b}$, V. L. RYKOV ${ }^{b}$, A. N. VASILIEV ${ }^{a, b}$ \\ ${ }^{a}$ State Research Center Institute for High Energy Physics, Protvino, 142284, Russia \\ ${ }^{b}$ National Research Nuclear University Moscow Engineering Physics Institute, Moscow, 115409, \\ Russia \\ E-mail: mochaloveihep.ru
}

Polarization phenomena in strong interactions and spin structure of hadrons still are not fully understood theoretically. The experimental data on spin-dependent interactions are also still scarce in some areas, for example, for antiproton-proton collisions.

Up to now, the E704 experiment at Fermilab has brought, probably, the largest sample of measured single-spin asymmetries for inclusive pion production in collisions of $200 \mathrm{GeV}$ polarized antiproton with unpolarized protons over a wide $x_{\mathrm{F}}$ range $\left(0.2<x_{\mathrm{F}}<0.9\right)$. The absolute values of observed asymmetries $A_{\mathrm{N}}$ for produced pions grow up as $\mathrm{x}_{\mathrm{F}}$ increases. For $\pi^{-}, A_{\mathrm{N}}$ is positive and grows up as large as $\sim 20 \%$. For $\pi^{0}$, it is also positive but smaller, just up to about $10 \%$. For $\pi^{+}, A_{\mathrm{N}}$ is large and negative, down to $-30 \%$.

The new SPASCHARM experiment at IHEP (Protvino, Russia) will study spin phenomena, using the polarized antiproton beam at the energy of $\sim 15 \mathrm{GeV}$. At the first stage of experiment, the spin effects in production of hadrons, consisting of light $u$-, $d$-, and $s$-quarks, will be studied. Single-spin asymmetry can be measured for tens of reactions. Polarization effects in hyperon and vector meson production will be explored as well. The second stage of the experiment has the goal to measure singlespin effects in charmonium production as well as carry out the double-spin asymmetry measurements in order to evaluate the contribution of gluon polarization into the spin of proton at large $x_{\mathrm{F}}$.

The PANDA experiment proposed at FAIR facility will provide the opportunity for measuring the polarization of strange and single-charmed hyperons in final states of antiproton-proton annihilation.

XXII International Baldin Seminar on High Energy Physics Problems

15-20 September 2014

JINR, Dubna, Russia

${ }^{1}$ Speaker 


\section{Introduction}

The interest in studying polarization phenomena in strong interactions arises from the study of spin structure of hadrons through interactions of constituent partons having non-zero spin. Numerous experimental results provided clear evidences that spin effects are significant. In perturbation theory of QCD at the leading twist, the transverse single-spin asymmetries are predicted close to zero and vanishing as energy increases [1]. In recent years, there has been a considerable progress in the experimental study of spin effects at high energies. It was found that single-spin asymmetries do not vary much in a wide energy range. This is illustrated by comparing the experimental data in Fig. 1 and 2 for reaction $p_{\uparrow} p \rightarrow \pi^{0} X$. The $A_{\mathrm{N}}$ asymmetry in Fig. 1 is from the fixed-target PROZA experiment at IHEP (Protvino) for the beam energy of 50 $\mathrm{GeV}$, that is at $\sqrt{ }_{s}=9.8 \mathrm{GeV}$ [2]. The same asymmetry in Fig. 2 is from the collider experiment STAR for the much higher energy at $\sqrt{s}_{s}=500 \mathrm{GeV}$ [3]. Interestingly, the substantially non-zero $A_{\mathrm{N}}$ has been found also in $\pi^{-} p_{\uparrow}$ collisions in the beam ${ }^{2}$ fragmentation region [4]. So far, there is still no theory, providing a complete and consistent description of polarization phenomena in strong interactions.

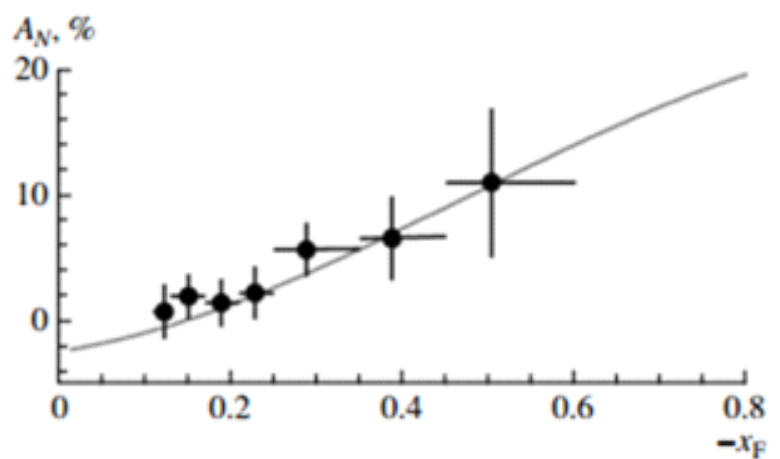

Fig. 1 The asymmetry $A_{\mathrm{N}}$ in reaction $p+p_{\uparrow} \rightarrow \pi^{0}+X$ in the polarized proton fragmentation region at beam energy 50 $\mathrm{GeV}[2]$.

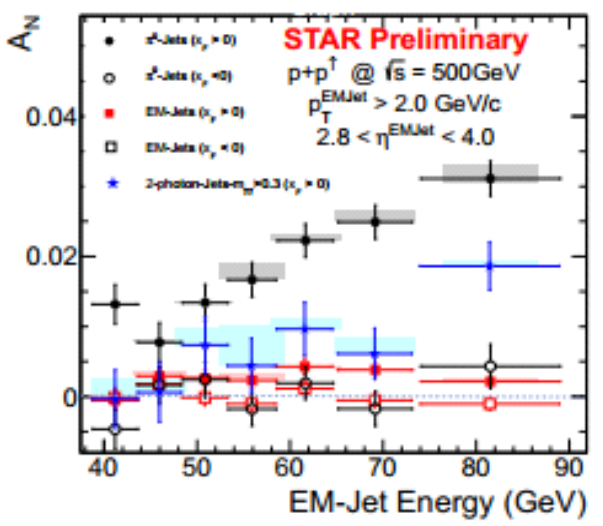

Fig 2: $A_{\mathrm{N}}$ for EM-jets vs. energy for isolated $\pi^{0}, 2$-photon EM-jets that fall outside $\pi^{0}$ mass definition, and for EM-jets containing $N_{\text {photons }}>2[3]$

\section{Polarization physics with antiprotons}

The other side of the 'coin' under discussion here are the experiments with antiprotons. Since the discovery of antimatter in $1930^{\text {th }}$, it always has been and continues to be the subject of a great attraction, first of all, for physicists and philosophers as well as for people outside of scientific communities such as writers, artists and many others. For physicist, the antiprotons are of a particular interest because they are the partners of protons which are a) the only absolutely

\footnotetext{
${ }^{2}$ Which was, obviously, unpolarized
} 
stable hadrons abundant and effectively "free" in the matter around us, and b) charged and thus easy to manipulate: accelerate, make proton beams and so forth ${ }^{4}$. This means that, as soon as antiprotons are available, virtually all of them could be utilized for studying the process of $\bar{p} p$ annihilation from the very pure charge-neutral ( $C$-neutral) $\bar{p} p$-state into the wide variety of final quantum states. In collisions of unpolarized protons and antiprotons, the initial states are $C P$ neutral as well. However, a $C P$-violation in annihilation process might potentially break the $C P$ neutrality of the final states. Measuring the polarization of particles, such as hyperons, in the final states may help checking the $C P$ - neutrality of final states, i.e. $C P$ - conservation in $\bar{p} p$ annihilation.

An annihilation of initially polarized protons and antiprotons brings the new quality into the study. With the polarized $p$ and $\bar{p}$, the initial state, generally, is not $C P$-neutral any more. But, with an ability of changing polarizations of the both, $p$ and $\bar{p}$, the $C P$-conjugate initial state can also be prepared. The comparison of characteristics of final states originated from two $C P$ conjugate initial states might potentially provide evidences for $C P$-violation in the primary process of $p \bar{p}$-annihilation. To the large extent, this is similar to the only, so far, observation at RHIC in BNL of direct spatial parity violation ( $P$-violation) in polarized proton collisions [5] (Fig.3). However, such a model independent test of $C P$-violation is impossible with two polarized proton beams only because the $C P$-conjugate initial state would require the collision of two polarized antiproton beams which are not available at RHIC or anywhere else.

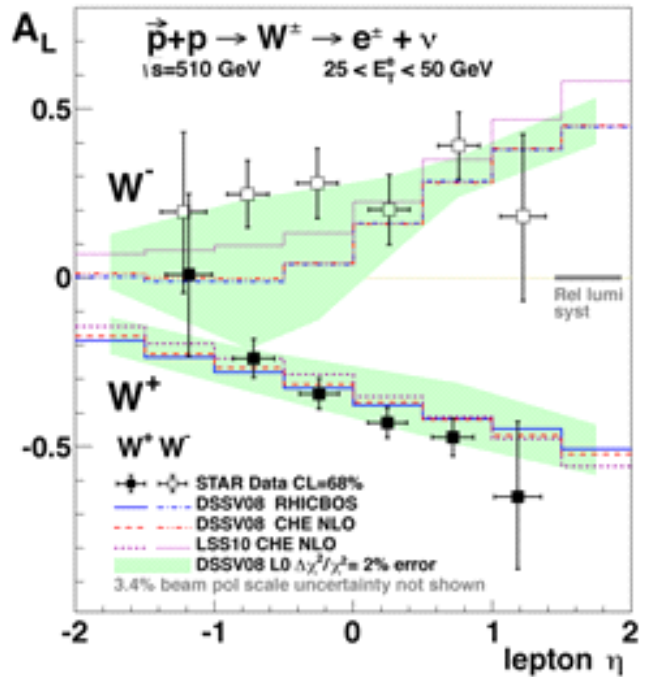

Fig. 3. The asymmetry in the production cross section for $W^{ \pm}$bosons in proton collisions with a positive (negative) proton beam helicity, $\sigma_{+}(\sigma)$, defined as $A_{L}=\left(\sigma_{+}-\sigma_{-}\right) /\left(\sigma_{+}+\sigma_{-}\right)$is shown as a function of the $W$ boson's decay lepton pseudorapidity. The filled (open) points correspond to the $W^{+}\left(W^{-}\right)$asymmetries, in comparison to predictions from different polarized parton distribution functions

In $p p$-collisions at low energies, the $q g$ - and $g g$-processes dominate in production of $c \bar{c}$ and $s \bar{s}$-pairs. An important feature of antiproton-proton reactions is the direct access to $q \bar{q}$ interactions via production of charmonium states.

\footnotetext{
${ }^{3}$ In hydrogen atoms, they are just weakly bound to atomic electrons through electromagnetic interactions.

${ }^{4}$ The only other particles with many similar properties, listed above, are electrons. But electrons and their antiparticles, positrons, do not participate in strong interactions.
} 
In general, virtually any experimental data for $p \bar{p}$-reactions with polarization are of the great interest just because such data are still very scarce. So far, the most comprehensive experimental study in this area has been performed in Fermilab experiment E704 [6-8]. The polarized proton and antiproton beams of momentum $p=200 \mathrm{GeV} / c$ have been formed from the parity-violating decays of $\Lambda$-hyperons [9]. The large asymmetries $A_{\mathrm{N}}$ in pion production at forward region have been observed as in $p_{t} p$-reaction as well as in $\bar{p}_{t} p$-collisions with transversely polarized protons or antiprotons of the beam. The results are shown side-by-side in Fig.4 [6] and Fig. 5 [7]. In $p_{t} p$ - as well as in $\bar{p}_{t} p$-collisions, the absolute values of observed asymmetries $A_{\mathrm{N}}$ for produced pions grow up as $x_{\mathrm{F}}$ increases. In $\bar{p}_{t} p$-collisions for $\pi^{-}, A_{\mathrm{N}}$ is positive and grows up as large as $\sim 20 \%$. For $\pi^{0}$, it is also positive but smaller, just up to about $10 \%$. For $\pi^{+}, A_{\mathrm{N}}$ is the largest and negative, down to $-30 \%$. It worth noting that, for charged pions, the asymmetries $A_{\mathrm{N}}$ are of opposite sign for polarized proton and antiproton beams while $A_{\mathrm{N}}$ for $\pi^{0}$ is of the same sign.

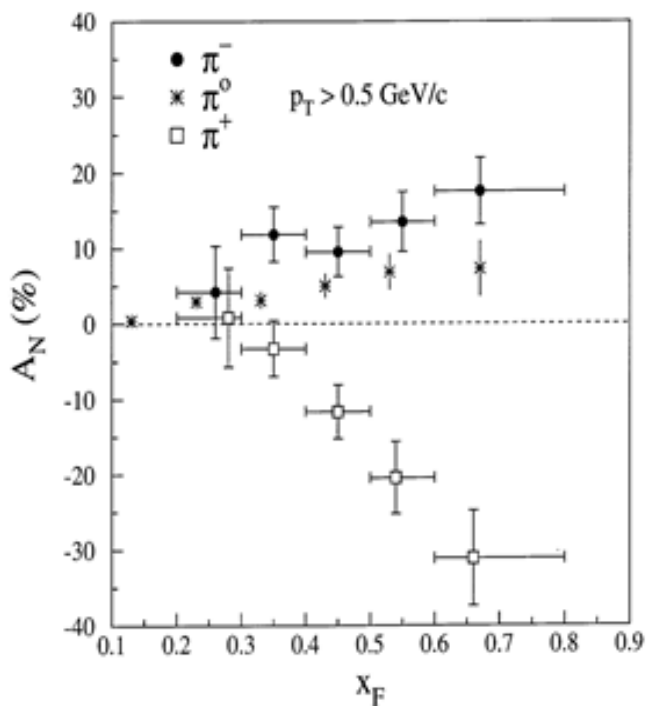

Fig. 4. $A_{\mathrm{N}}$ data [6] in $\bar{p}_{\uparrow} p$-collisions as function of $x_{\mathrm{F}}$ for $\pi^{-}$and $\pi^{+}$for $p_{T}>0.5 \mathrm{GeV} / \mathrm{c} . A_{\mathrm{N}}$ data for $\pi^{0}$ in a similar $p_{\mathrm{T}}$ range are also shown [7]. The first $\pi^{-}$and $\pi^{+}$data points are offset by -0.01 and $+0.01 x_{\mathrm{F}}$ units, respectively

For more than 20 years, the E704 results along with the other available data from polarization experiments have been the focus of many theoretical studies (see, for example, Refs. [10-17]). Still, neither one can currently be named as a full and consistent theory for polarization phenomena in strong interactions, although some of them, like the model of effective color field [18], pretend on describing the whole range of polarization effects. Yet, it needs to be verified and confirmed with the new experimental data, and the contribution of two 
new projects with antiprotons, which are currently under development, would be of an invaluable importance.

\section{Study of polarization effects with antiprotons at SPASCHARM experiment}

The SPASCHARM experiment [19] is currently under preparation at the IHEP (Protvino) proton synchrotron U-70. At its first stage, the SPASCHARM will measure single-spin asymmetries in collisions of unpolarized negative beam with polarized proton target. The composition of the beam is predominantly the negatively pions with the sizeable admixtures of kaons and antiprotons. The presence of antiprotons provides the opportunity for measuring single-spin asymmetries in annihilation processes already at the first stage of SPASCHARM project. The expected statistics for some selected reaction are presented in Table.1.

Table.1 Expected statistics for selected reactions in $\bar{p} p_{\uparrow}$-annihilation for one month of data taking at the $1^{\text {st }}$ stage of SPASCHARM experiment

\begin{tabular}{|l|l|l|l|l|l|l|l|}
\hline № & Particle & $\mathrm{N}_{\mathrm{EV}}$ & $\mathrm{S} / \mathrm{B}$ & № & Particle & $\mathrm{N}_{\mathrm{EV}}$ & $\mathrm{S} / \mathrm{B}$ \\
\hline 1 & $\pi^{+}$ & $2.1 \cdot 10^{8}$ & & 7 & $n$ & $1.6 \cdot 10^{7}$ & \\
\hline 2 & $\pi^{-}$ & $2.6 \cdot 10^{8}$ & & 8 & $\bar{n}$ & $1.4 \cdot 10^{8}$ & \\
\hline 3 & $K^{+}$ & $1.7 \cdot 10^{7}$ & & 9 & $\bar{\Lambda} \rightarrow \bar{p} \pi^{+}$ & $2.1 \cdot 10^{6}$ & 10 \\
\hline 4 & $K^{-}$ & $2.2 \cdot 10^{7}$ & & 10 & $\bar{\Lambda} \rightarrow \bar{n} \pi^{0}$ & $1.1 \cdot 10^{6}$ & 0.13 \\
\hline 5 & $p$ & $1.6 \cdot 10^{7}$ & & 11 & $\bar{\Delta}^{--} \rightarrow \bar{p} \pi^{-}$ & $4.2 \cdot 10^{7}$ & 0.14 \\
\hline 6 & $\bar{p}$ & $1.8 \cdot 10^{8}$ & & 12 & $\Xi^{-} \rightarrow \Lambda \pi^{-}$ & $1.0 \cdot 10^{5}$ & 10 \\
\hline
\end{tabular}

It will also be possible to measure spin asymmetries in inclusive production of $\eta$ - and $\phi$ mesons (Fig. 6 right). The latter one is of a particular interest due to its $\bar{s}$ quark-pair composition.
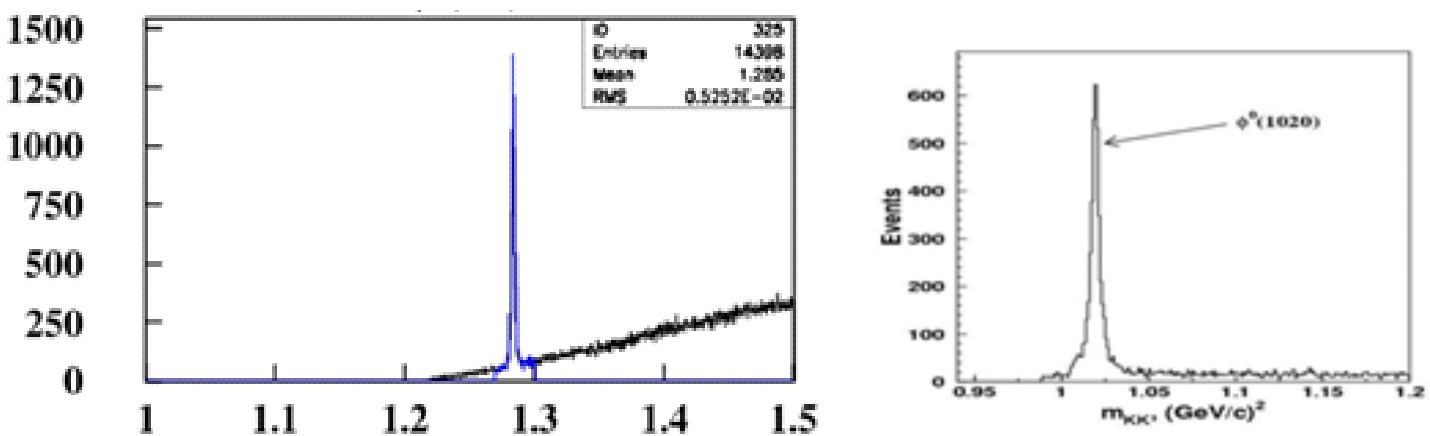

Fig. 6. The effective mass distributions in productions of $\Xi \rightarrow \Lambda \pi^{-}$(left) and $\phi$-meson by $34 \mathrm{GeV}$ antiprotons 
At the $2^{\text {nd }}$ stage of SPASCHARM experiment, the polarized proton and antiproton beams will be available for interactions with the unpolarized and polarized targets. The polarized protons and antiprotons will be produced from the parity-violating decays of $\Lambda$ - and $\bar{\Lambda}$-hyperons [20, 21, 9]. By design, the SPASCHARM 12-20 GeV antiproton beam [19] of total intensity up to $\sim 4 \cdot 10^{5}$ antiproton per 9-second U-70 cycle will be split for physics analysis, using beamtagging information, into three sub-samples with the average transverse polarization of $-40 \%, 0$, and $+40 \%$. The new polarized beam at IHEP will allow measuring the spin asymmetries in tens of reactions in the polarized particle fragmentation region at various targets. The latter is important for verifying the predictions of the effective color model [18] for single-spin asymmetry dependences on the target's atomic number. The examples of predictions for reaction are shown in Fig.7.

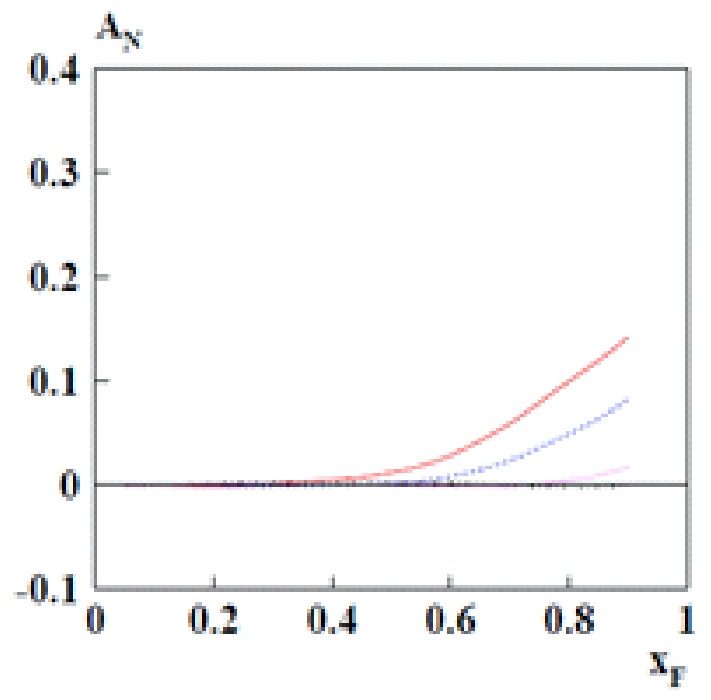

Fig. 7 The predicted dependences of $A_{\mathrm{N}}\left(x_{\mathrm{F}}\right)$ in reaction $\bar{p}_{\uparrow} A \rightarrow \pi^{-} X$ at $\sqrt{s}_{N N}=5.48 \mathrm{GeV} /$ nucleon for four different nuclei (from top to bottom): $\mathrm{C}, \mathrm{Cu}, \mathrm{Sn}$ and $\mathrm{Pb}[22]$

Among other things, we plan measuring at SPASCHARM the spin-density matrix for produced vector meson as well as polarization parameters for hyperons in final states (polarization, depolarization, etc.).

The single- and double-spin asymmetries in Charmonium production will be measured at SPASCHARM with polarized proton and antiproton beams. The quark-antiquark annihilation channel is responsible for about $95 \%$ of charmonium production cross section in $\bar{p} p$-collisions, while $g g$ - and $q g$-interactions dominate in $p p$-collisions. The studies of charmonium production with the both, polarized proton and antiproton beams, provide an unique opportunity for separating the contributions of different parton interaction channels into charmonium production cross section and spin asymmetries.

It worth mentioning that, at the both stages of SPASCHARM experiment, the simultaneous measurements of various spin-dependent physical observables will be available: SSA, hyperon polarization, spin density matrix elements and alignment for vector mesons, spin transfer parameters. The large solid angle acceptance of SPASCHARM experiment allows to 
carry out measurements at a wide kinematic range $\left(0<x_{\mathrm{F}}<1,0.5<p_{\mathrm{T}}<3 \mathrm{GeV} / c\right.$, beam energy from 12 to $50 \mathrm{GeV}$ ) while the full $2 \pi$ coverage in azimuth helps to minimize the systematic errors in spin asymmetries.

\section{Study of hyperon polarization at PANDA}

The main objectives of PANDA experiment [23-25] under construction at the FAIR facility (Darmstadt, Germany) are the search and study of properties of exotic states, such as glueballs, hybrids, molecules, XYZ-states, etc. It will operate at the High Energy Storage Ring (HESR), utilizing the high-intensity and low emittance antiproton beam ${ }^{5}$ of momenta from 1.5 to $15 \mathrm{GeV} / c$. Regarding specifically the $\bar{p} p$-collisions, PANDA will cover center of mass energies of up to $\sqrt{ }_{s}=5.5 \mathrm{GeV}$ but overlap with the range explored by LEAR experiment [26] of CERN at the low energy end. The list of topics to be addressed by PANDA experiment includes a wide range of issues in hadron spectroscopy and hadron dynamics.

The study of hyperon and antihyperon production represents a natural part of PANDA's focus on exploration of baryonic final states. The parity-violating weak decays of hyperons provide the powerful tool for accessing the final state baryon's polarization. In the simplest cases, such as $\Lambda \rightarrow p \pi^{-}$and $\bar{\Lambda} \rightarrow \bar{p} \pi^{+}$decays, the hyperon polarization is derived at one step from the measured anisotropy of decay proton or antiproton emission. Quite comprehensive studies of reaction $\bar{p} p \rightarrow \bar{\Lambda} \Lambda$ have been performed at LEAR [26]. At PANDA experiment, with its close to $4 \pi$-acceptance and excellent detection capabilities for charged and neutral particles with precise measurements of their characteristics, the more complex analysis of cascade decays would be feasible. As an example, Fig. 8 illustrates the multistage decay of $\Xi$-hyperon and its antiparticle: $\bar{\Xi}^{+} \rightarrow \bar{\Lambda} \pi^{+}, \bar{\Lambda} \rightarrow \bar{p} \pi^{+}$.

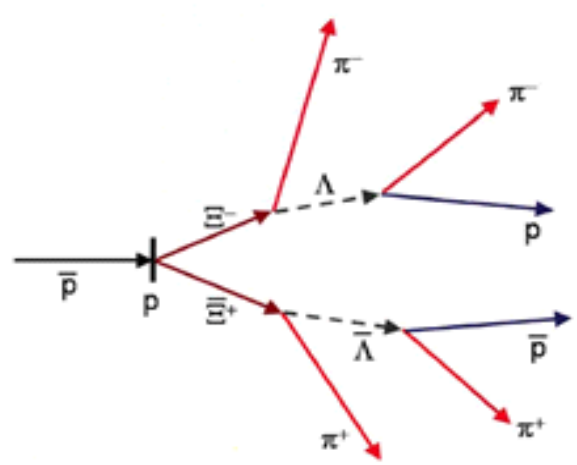

Fig. 8 Schematic illustration for the topology of $\bar{p} p \rightarrow \bar{\Xi}^{+} \Xi^{-}$production and decay chain

For the events with hyperon-antihyperon pairs in the final state, the hyperon-antihyperon spin correlation parameters will be measured which potentially might provide an access to the spin structure of the produced $s \bar{s}$-pairs. So far, there are no experimental data on angular distributions, polarization, or spin correlations in any of the following reaction: $\bar{p} p \rightarrow \bar{\Xi}^{+} \Xi^{-}$,

${ }^{5}$ Unpolarized 
$\bar{\Omega}^{+} \Omega^{-}, \bar{\Lambda}^{-} \Lambda^{+}$. The program for studying spin effects in hyperon-antihyperon production at PANDA has been presented in [27]. The estimates of event rate for some reactions are shown in Table 2.

Table 2. The estimates for production rates of various hyperon channels at PANDA experiment [27-29]

\begin{tabular}{|c|c|c|c|c|c|}
\hline $\begin{array}{c}\text { Beam Momentum } \\
(\mathrm{GeV} / \mathrm{c})\end{array}$ & Reeaction & $\sigma(\mu \mathrm{b})$ & Eff $(\%)$ & Decay & $\begin{array}{c}\text { Rate at } \\
2 \cdot 10^{32} \mathrm{~cm}^{-2} \mathrm{~s}^{-1}\end{array}$ \\
\hline 1,64 & $\bar{p} p \rightarrow \bar{\Lambda} \Lambda$ & 64 & 11 & $\Lambda \rightarrow p \pi^{-}$ & $580 \mathrm{~s}^{-1}$ \\
\hline 4 & $\bar{p} p \rightarrow \bar{\Lambda} \Sigma^{0}$ & 40 & 31 & $\Sigma^{0} \rightarrow \Lambda \gamma$ & $600 \mathrm{~s}^{-1}$ \\
\hline 4 & $\bar{p} p \rightarrow \bar{\Xi}^{+} \Xi^{-}$ & $\sim 2$ & 19 & $\Xi^{-} \rightarrow \Lambda \pi^{-}$ & $30 \mathrm{~s}^{-1}$ \\
\hline 12 & $\bar{p} p \rightarrow \bar{\Omega}^{+} \Omega^{-}$ & $\sim 0.002$ & $\sim 30$ & $\Omega \rightarrow \Lambda K^{-}$ & $\approx 80 \mathrm{~h}^{-1}$ \\
\hline 12 & $\bar{p} p \rightarrow \bar{\Lambda}_{c}^{-} \Lambda^{+}$ & $\sim 0.1 \mathrm{nb}$ & $\sim 35$ & $\Lambda_{c} \rightarrow \Lambda \pi^{+}$ & $\approx 25 \mathrm{~d}^{-1}$ \\
\hline
\end{tabular}

\section{Conclusions}

Polarization phenomena in reactions with antiprotons represent the whole rich world for exploration with the goal of better understanding the, sometimes, seemingly subtle but, in fact, still very important role of spin in strong interactions. The two new experiments under construction now, which have been briefly described in this report, will represent the new significant steps is such explorations.

The SPASCHARM experiment at U-70 accelerator of IHEP (Protvino, Russia) is designed to utilize the new polarized proton and antiproton beams for measuring the single- and doublespin asymmetries in a wide variety of reactions with detection of the both, charged and neutral particles in the final states, originated from proton-proton and antiproton-proton collisions at the beam momenta of $\sim 12-50 \mathrm{GeV} / c$. The single- and double-spin asymmetries in Charmonium production will be measured at SPASCHARM with polarized proton and antiproton beams. The quark-antiquark annihilation channel is responsible for about $95 \%$ of charmonium production cross section in $\bar{p} p$-collisions, while $g g$ - and $q g$-interactions dominate in $p p$-collisions. The coming studies of charmonium production with polarized protons and antiprotons are anticipated to be the source of unique data for separating the contributions of different parton interaction channels into charmonium production.

The PANDA experiment at FAIR facility (Darmstadt, Germany) will operate at the highintensity and low emittance antiproton beam of HESR storage ring. The PANDA's close to $4 \pi$ acceptance and its excellent detection capabilities for charged and neutral particles with precise measurements of their characteristics make it suitable for the full reconstruction of the most complex cascade hyperon decays in the final state and, eventually, the measurement of hyperon polarizations in final states and their correlations. 
These two, to some extent, complimentary new experimental programs will undoubtedly generate by their results quite a bit of excitement in the particle physics community in the coming years.

\section{Acknowledment}

We thank the IHEP management and the IHEP Accelerator, Beam and Electronics departments for their invaluable contribution in construction and commissioning of the setup at the beam, and for their support of the SPASCHARM program. We also thank V. Abramov for invaluable discussion on the spin-physics theoretical approaches. This work was supported in part by the RFBR grants \# 12-02-00737-a and \# 15-02-01675-a.

\section{References}

[1] G. L. Kane, J. Pumplin and W. Repko, Transverse Quark Polarization in Large p(T) Reactions, $e^{+} e^{-}$ Jets, and Leptoproduction: A Test of QCD, Phys. Rev. Lett. 42, 1689 (1978).

[2] V.V. Abramov et al., Analyzing Power in the Reaction $p+p \rightarrow \pi^{0}+X$ in the polarized-target fragmentation region at an energy of $50 \mathrm{GeV}$, Phys.Atom.Nucl. 77, 595 (2014).

[3] M. Mondal (for STAR Collabotration), Measurement of the Transverse Single-Spin Asymmetries for $\pi^{0}$ and Jet-like Events at Forward Rapidities at STAR in $p+p$ Collisions at $\sqrt{ } s=500 \mathrm{GeV}$, PoS (DIS2014) 216 (2014) [nucl-ex/1407.3715].

[4] V.V. Mochalov et al, Measurement of the single-spin asymmetry in the reaction $\pi^{-} d_{\uparrow} \rightarrow \pi^{0} X$ in the beam-fragmentation region at 40-GeV and $p_{\mathrm{T}}$ of up to 2-GeV/c, Phys.Atom.Nucl. 73, 2017 (2010).

[5] L. Adamczyk et al. (STAR Collaboration), Measurement of longitudinal spin asymmetries for weak boson production in polarized proton-proton collisions at RHIC, Phys. Rev. Lett. 113, 072301 (2014) [nucl-ex/1404.6880].

[6] A. Bravar, et al. (E704 Collaboration), Single spin asymmetries in inclusive charged pion production by transversely polarized anti-protons, Phys. Rev. Lett. 77, 2626 (1996).

[7] D. L. Adams et al. (E704 Collaboration), Analyzing power in inclusive $\pi^{+}$and $\pi^{-}$- production at high $x_{\mathrm{F}}$ with a 200-GeV polarized proton beam, Phys. Lett. B 264, 462 (1991).

[8] D. L. Adams, et al. (E704 Collaboration), Comparison of spin asymmetries and cross-sections in pi0 production by 200-GeV polarized anti-protons and protons, Phys. Lett. B 261, 201 (1991).

[9] D. P. Grosnick et al., The Design and Performance of the Fnal High-energy Polarized Beam Facility, Nucl.Instrum.Meth. A 290, 269 (1990).

[10] D. Sivers, Single Spin Production Asymmetries from the Hard Scattering of Point-Like Constituents, Phys. Rev. D 41, 83 (1990); Hard scattering scaling laws for single spin production asymmetries, Phys. Rev. D 43, 261 (1991).

[11] J. Collins, Fragmentation of transversely polarized quarks probed in transverse momentum distributions, Nucl. Phys. B 396, 161 (1993). 
[12] U. D’Alesio, F. Murgia, and C. Pisano, Azimuthal asymmetries for hadron distributions inside a jet in hadronic collisions, Phys. Rev. D 83, 034021 (2011) [hep-ph/1011.2692].

[13] M. Anselmino, M. Boglione, U. D’Alesio, S. Melis, F. Murgia, and A. Prokudin, Sivers effect and the single spin asymmetry $A_{\mathrm{N}}$ in $p_{\uparrow} p \rightarrow h X$ processes Phys. Rev. D 88, 054023 (2013) [hep$\mathrm{ph} / 1304.7691]$.

[14] Z.-B. Kang and F. Yuan, Single Spin Asymmetry Scaling in the Forward Rapidity Region at RHIC, Phys. Rev. D 84, 034019 (2011) [hep-ph/ 1106.1375].

[15] A. Efremov and O. Teryaev, On Spin Effects in Quantum Chromodynamics Yad. Fiz. 36, 242 (1982) [Sov. J. Nucl. Phys. 36, 140 (1982)]; QCD Asymmetry and Polarized Hadron Structure Functions, Phys. Lett. B 150, 383 (1984).

[16] J. Qiu and G. Sterman, Single transverse spin asymmetries in hadronic pion production, Phys. Rev. D 59, 014004 (1998) [hep-ph/9806356].

[17] K. Kanazawa and Y. Koike, A phenomenological study on single transverse-spin asymmetry for inclusive light-hadron productions at RHIC Phys. Rev. D 83, 114024 (2011) [hep-ph/1104.0117].

[18] V.V. Abramov, Phenomenology of single-spin effects in hadron production at high energies Phys.Atom.Nucl. 72, 2009 (1872) [Yad.Fiz. 72, 1933 (2009)].

[19] V. Mochalov, Spin physics at IHEP, Phys.Part.Nucl. 44, issue 6, 930 (2013).

[20] O. E. Overseth, Proc. of National Accelerator Laboratory 1969 Summer Study, Report SS-118 2250, Available in eConf C69-06-09, 005 (1969).

[21] O. E. Overseth and J. Sandweiss, Proc. of National Accelerator Laboratory 1969 Summer Study, Report SS-120 2250; Available in eConf C69-06-09, 006 (1969).

[22] V. Abramov, private communication.

[23] Physics Performance Report for PANDA: Strong Interaction Studies with Antiprotons -e-print arXiv: hep-ex 0903.3905.

[24] A. Gillitzer, Strong interaction physics with antiproton-induced reactions at PANDA Hyperfine Interact., 229, issue 1-3, 21 (2014).

[25] V. Mochalov, Physics with antiprotons at PANDA, Nucl.Phys.Proc.Suppl. 245, 124 (2013).

[26] Paschke, K.D. et al. Experimental determination of the complete spin structure for anti-pp $\rightarrow$ anti$\Lambda \Lambda$ at $p($ anti $p)=1.637 \mathrm{GeV} / \mathrm{c}$. Phys. Rev. C 74, 015206 (2006).

[27] K. Schönning et al., Spin observables in antihyperon-hyperon production with PANDA, J.Phys.Conf.Ser. 556, issue 1012007 (2014).

[28] E. Thomė, Multi-strange and charmed antihyperon-hyperon physics for PANDA. Doctoral thesis Uppsala Universitet (2012).

[29] S. Grape, Studies of PWO Crystals and Simulations of the $\bar{p} p \rightarrow \bar{\Lambda} \Lambda, \Lambda \Sigma^{0}$ Reactions for the PANDA experiment Ph.D. Thesis, Uppsala University (2009). 